\title{
An Introduction to Statistical Mechanics and Thermodynamics
}

\author{
Robert H. Swendsen
}




\section{Contents}

Preface $\quad$ xV

1 Introduction 1

1.1 Thermal Physics 1

1.2 What are the Questions?

1.3 History 2

1.4 Basic Concepts and Assumptions 4

1.5 Road Map 5

Part I Entropy

2 The Classical Ideal Gas 11

2.1 Ideal Gas $\quad 11$

2.2 Phase Space of a Classical Gas 12

2.3 Distinguishability 12

2.4 Probability Theory 13

2.5 Boltzmann's Definition of the Entropy 13

$2.6 S=k \log W \quad 14$

2.7 Independence of Positions and Momenta 14

2.8 Road Map for Part I $\quad 15$

3 Discrete Probability Theory 16

3.1 What is Probability? 16

3.2 Discrete Random Variables and Probabilities $\quad 17$

3.3 Probability Theory for Multiple Random Variables 18

3.4 Random Numbers and Functions of Random Variables 20

3.5 Mean, Variance, and Standard Deviation 22

3.6 Correlation Functions 23

3.7 Sets of Independent Random Numbers $\quad 24$

3.8 Binomial Distribution $\quad 25$

3.9 Gaussian Approximation to the Binomial Distribution 27

3.10 A Digression on Gaussian Integrals 28

3.11 Stirling's Approximation for $N$ ! 29

3.12 Binomial Distribution with Stirling's Approximation 32

3.13 Problems 33

4 The Classical Ideal Gas: Configurational Entropy 40

4.1 Separation of Entropy into Two Parts 40

4.2 Distribution of Particles between Two Subsystems 41

4.3 Consequences of the Binomial Distribution 42

4.4 Actual Number versus Average Number $\quad 43$ 
viii Contents

4.5 The 'Thermodynamic Limit' 44

4.6 Probability and Entropy 44

4.7 An Analytic Approximation for the Configurational 46

5 Continuous Random Numbers 47

5.1 Continuous Dice and Probability Densities 47

5.2 Probability Densities 48

5.3 Dirac Delta Functions $\quad 50$

5.4 Transformations of Continuous Random Variables 53

5.5 Bayes' Theorem $\quad 55$

$\begin{array}{ll}5.6 & \text { Problems } \\ \end{array}$

6 The Classical Ideal Gas: Energy-Dependence
of Entropy

6.1 Distribution for the Energy between Two Subsystems 62

6.2 Evaluation of $\Omega_{E} \quad 64$

6.3 Probability Distribution for Large $N$

6.4 The Logarithm of the Probability Distribution and the

7 Classical Gases: Ideal and Otherwise 71

7.1 Entropy of a Composite System of Classical Ideal Gases 71

$\begin{array}{ll}7.2 & \text { Equilibrium Conditions for the Ideal Gas } \\ 7.32\end{array}$

7.3 The Volume-Dependence of the Entropy 74

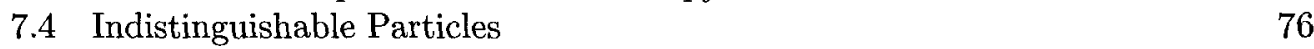

7.5 Entropy of a Composite System of Interacting Particles 78

7.6 The Second Law of Thermodynamics 83

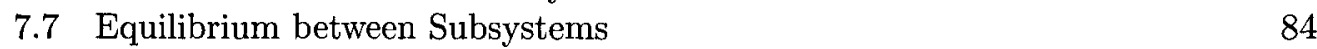

7.8 The Zeroth Law of Thermodynamics 85

$\begin{array}{lll}7.9 & \text { Problems } & 86\end{array}$

8 Temperature, Pressure, Chemical Potential,
and All That

8.1 Thermal Equilibrium $\quad 88$

$\begin{array}{ll}8.2 \text { What do we Mean by 'Temperature'? } & 89\end{array}$

$\begin{array}{ll}8.3 \text { Derivation of the Ideal Gas Law } & 90\end{array}$

8.4 Temperature Scales 93

8.5 The Pressure and the Entropy 94

8.6 The Temperature and the Entropy 95

8.7 The Entropy and the Chemical Potential 95

8.8 The Fundamental Relation and Equations of State 96

8.9 The Differential Form of the Fundamental Relation 97

$\begin{array}{ll}\text { 8.10 Thermometers and Pressure Gauges } & 97\end{array}$

$\begin{array}{ll}8.11 \text { Reservoirs } & 97\end{array}$

$\begin{array}{ll}8.12 \text { Problems } & 98\end{array}$ 
Part II Thermodynamics

9 The Postulates and Laws of Thermodynamics $\quad 101$

$\begin{array}{lll}9.1 & \text { Thermal Physics } & 101\end{array}$

9.2 Microscopic and Macroscopic States 103

$\begin{array}{ll}\text { 9.3 Macroscopic Equilibrium States } & 103\end{array}$

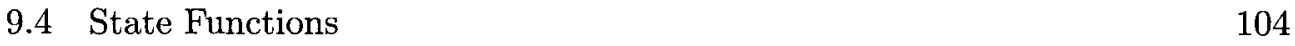

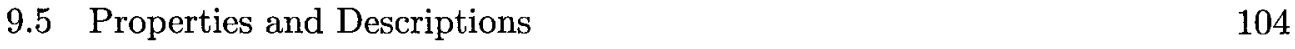

$\begin{array}{ll}\text { 9.6 Postulates of Thermodynamics } & 104\end{array}$

$\begin{array}{ll}\text { 9.7 The Laws of Thermodynamics } & 107\end{array}$

10 Perturbations of Thermodynamic State Functions 109

10.1 Small Changes in State Functions 109

10.2 Conservation of Energy $\quad 110$

10.3 Mathematical Digression on Exact and Inexact Differentials 110

10.4 Conservation of Energy Revisited $\quad 113$

10.5 An Equation to Remember 114

$\begin{array}{ll}10.6 \text { Problems } & 115\end{array}$

11 Thermodynamic Processes 116

11.1 Irreversible, Reversible, and Quasi-Static Processes 116

$\begin{array}{ll}11.2 \text { Heat Engines } & 117\end{array}$

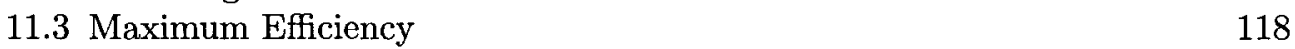

$\begin{array}{ll}11.4 \text { Refrigerators and Air Conditioners } & 119\end{array}$

11.5 Heat Pumps $\quad 120$

11.6 The Carnot Cycle $\quad$ : 121

$\begin{array}{ll}11.7 \text { Problems } & 121\end{array}$

12 Thermodynamic Potentials 123

12.1 Mathematical digression: the Legendre Transform $\quad 123$

12.2 Helmholtz Free Energy $\quad 126$

$\begin{array}{ll}12.3 \text { Enthalpy } & 128\end{array}$

$\begin{array}{ll}12.4 \text { Gibbs Free Energy } & 129\end{array}$

12.5 Other Thermodynamic Potentials 130

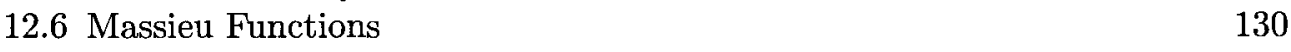

12.7 Summary of Legendre Transforms $\quad 130$

$\begin{array}{ll}12.8 \text { Problems } & 131\end{array}$

13 The Consequences of Extensivity 133

13.1 The Euler Equation $\quad 133$

13.2 The Gibbs-Duhem Relation 134

13.3 Reconstructing the Fundamental Relation $\quad 135$

$\begin{array}{ll}13.4 \text { Thermodynamic Potentials } & 137\end{array}$

14 Thermodynamic Identities 138

14.1 Small Changes and Partial Derivatives 138

14.2 A Warning about Partial Derivatives 138 
14.3 First and Second Derivatives $\quad 13 \mathrm{C}$

14.4 Standard Set of Second Derivatives 140

14.5 Maxwell Relations 141

14.6 Manipulating Partial Derivatives $\quad 143$

$\begin{array}{lll}14.7 & \text { Working with Jacobians } & 146\end{array}$

14.8 Examples of Identity Derivations 148

14.9 General Strategy $\quad 151$

14.10 Problems $\quad 152$

15 Extremum Principles $\quad 156$

$\begin{array}{lll}15.1 & \text { Energy Minimum Principle } & 156\end{array}$

15.2 Minimum Principle for the Helmholtz Free Energy $\ldots \ldots$

$\begin{array}{lll}15.3 & \text { Minimum Principle for the Enthalpy } & 162\end{array}$

15.4 Minimum Principle for the Gibbs Free Energy : $\quad 163$

$\begin{array}{lll}15.5 & \text { Exergy } & 164\end{array}$

15.6 Maximum Principle for Massieu Functions 165

$\begin{array}{lll}15.7 & \text { Summary } & 165\end{array}$

$\begin{array}{ll}15.8 \text { Problems, } & 165\end{array}$

16 Stability Conditions 167

$\begin{array}{lll}16.1 & \text { Intrinsic Stability } & 167\end{array}$

16.2 Stability Criteria based on the Energy Minimum Principle 168

16.3 Stability Criteria based on the Helmholtz Free
Energy Minimum Principle

16.4 Stability Criteria based on the Enthalpy Minimization

16.5 Inequalities for Compressibilities and Specific Heats 172

$\begin{array}{lll}16.6 & \text { Other Stability Criteria } & 173\end{array}$

$\begin{array}{lll}16.7 & \text { Problems } & 175\end{array}$

17 Phase Transitions $\quad 177$

$\begin{array}{lll}17.1 & \text { The van der Waals Fluid } & 178\end{array}$

$\begin{array}{lll}17.2 & \text { Derivation of the van der Waals Equation } & 178\end{array}$

$\begin{array}{ll}17.3 \text { Behavior of the van der Waals Fluid } & 179\end{array}$

$\begin{array}{ll}17.4 \text { Instabilities: } & 180\end{array}$

17.5 The Liquid-Gas Phase Transition 182

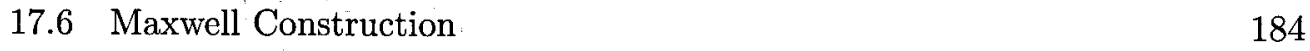

17.7 Coexistent Phases 184

17.8 Phase Diagram $\quad 185$

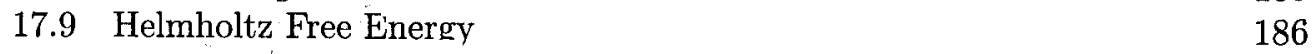

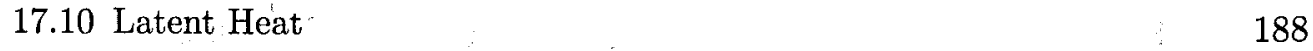

17.11 The Clausius-Clapeyron Equation $\quad 188$

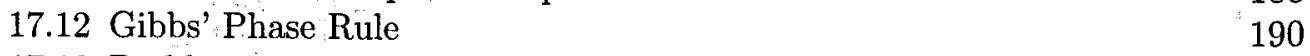

$\begin{array}{ll}17.13 \text { Problems } & 191\end{array}$ 
18 The Nernst Postulate: the Third Law of Thermodynamics

18.1 Classical Ideal Gas Violates the Nernst Postulate

18.2 Planck's Form of the Nernst Postulate

18.3 Consequences of the Nernst Postulate 195

18.4 Coefficient of Thermal Expansion at Low Temperatures 196

18.5 Summary and Signposts

Part III Classical Statistical Mechanics

19 Ensembles in Classical Statistical Mechanics": 201

19.1 Microcanonical Ensemble. 202

19.2 Molecular Dynamics: Computer Simulations 202

19.3 Canonical Ensemble . . 204

19.4 The Partition Function as an Integral over Phase Space $\quad 207$

19.5 The Liouville Theorem 207

19.6 Consequences of the Canonical Distribution.. 209

19.7 The Helmholtz Free Energy $\quad 210$

19.8 Thermodynamic Identities $\quad 211$

19.9 Beyond Thermodynamic Identities $\quad 212$

19.10 Integration over the Momenta 213

19.11 Monte Carlo Computer Simulations 214

19.12 Factorization of the Partition Function: the Best Trick in Statistical Mechanics 217

19.13 Simple Harmonic Oscillator $\quad 218$

19.14 Problems 220

20 Classical Ensembles: Grand and Otherwise 227

20.1 Grand Canonical Ensemble 227

20.2 Grand Canonical Probability Distribution 228

20.3 Importance of the Grand Canonical Partition Function $\quad 230$

$20.4 \mathcal{Z}(T, V, \mu)$ for the Ideal Gas 231

20.5 Summary of the Most Important Ensembles 231

$\begin{array}{ll}20.6 & \text { Other Classical Ensembles } \\ 20.7 & 232\end{array}$

$\begin{array}{lll}20.7 & \text { Problems } & 232\end{array}$

21 Irreversibility 234

21.1 What Needs to be Explained? 234

21.2 Trivial Form of Irreversibility 235

$\begin{array}{lll}21.3 & \text { Boltzmann's H-Theorem } & 235\end{array}$

21.4 Loschmidt's Umkehreinwand 235

21.5 Zermelo's Wiederkehreinwand 236

21.6 Free Expansion of a Classical Ideal Gas $\quad 236$

21.7 Zermelo's Wiederkehreinwand Revisited 240

21.8 Loschmidt's Umkehreinwand Revisited 241

21.9 What is 'Equilibrium'? 242

21.10 Entropy $\quad 242$

21.11 Interacting Particles 243 
xii Contents

Part IV Quantum Statistical Mechanics

22 Quantum Ensembles 247

22.1 Basic Quantum Mechanics $\quad 248$

22.2 Energy Eigenstates 248

22.3 Many-Body Systems $\quad 251$

22.4 Two Types of Probability 252

22.5 The Density Matrix 254

22.6 The Uniqueness of the Ensemble $\quad 255$

22.7 The Quantum Microcanonical Ensemble 256

23 Quantum Canonical Ensemble 258

23.1 Derivation of the QM Canonical Ensemble 258

23.2 Thermal Averages and the Average Energy 260

23.3 The Quantum Mechanical Partition Function 260

23.4 The Quantum Mechanical Entropy 262

23.5 The Origin of the Third Law of Thermodynamics 264

23.6 Derivatives of Thermal Averages 266

23.7 Factorization of the Partition Function 266

$\begin{array}{lll}23.8 & \text { Special Systems } & 269\end{array}$

23.9 Two-Level Systems $\quad 269$

23.10 Simple Harmonic Oscillator $\quad 271$

23.11 Einstein Model of a Crystal 273

$\begin{array}{ll}23.12 \text { Problems } & 275\end{array}$

24 Black-Body Radiation $\quad 282$

24.1 Black Bodies $\quad 282$

24.2 Universal Frequency Spectrum 282

24.3 A Simple Model $\quad 283$

24.4 Two Types of Quantization 283

24.5 Black-Body Energy Spectrum $\quad 285$

24.6 Total Energy 288

24.7 Total Black-Body Radiation $\quad 289$

24.8 Significance of Black-Body Radiation 289

24.9 Problems $\quad 289$

25 The Harmonic Solid 291

25.1 Model of an Harmonic Solid 291

25.2 Normal Modes $\quad 292$

25.3 Transformation of the Energy 296

25.4 The Frequency Spectrum 298

25.5 The Energy in the Classical Model 299

25.6 The Quantum Harmonic Crystal 300

25.7 Debye Approximation $\quad 301$

25.8 Problems 306 
26.1 Single-Particle Quantum States 308

$\begin{array}{ll}26.2 \text { Density of Single-Particle States } & 310\end{array}$

26.3 Many-Particle Quantum States $\quad 311$

$\begin{array}{ll}26.4 \text { Quantum Canonical Ensemble } & 312\end{array}$

26.5 Grand Canonical Ensemble $\quad 312$

26.6 A New Notation for Energy Levels 313

26.7 Exchanging Sums and Products 315

26.8 Grand Canonical Partition Function for Independent

26.9 Distinguishable Quantum Particles $\quad 316$

26.10 Sneaky Derivation of $P V=N k_{B} T \quad 317$

26.11 Equations for $U=\langle E\rangle$ and $\langle N\rangle \quad 318$

$26.12\left\langle n_{\epsilon}\right\rangle$ for bosons $\quad 319$

$26.13\left\langle n_{\epsilon}\right\rangle$ for fermions $\quad 319$

26.14 Summary of Equations for Fermions and Bosons 320

26.15 Integral Form of Equations for $N$ and $U \quad 321$

26.16 Basic Strategy for Fermions and Bosons 322

$26.17 P=2 U / 3 V \quad 322$

26.18 Problems $\quad 324$

27 Bose-Einstein Statistics $\quad 326$

27.1 Basic Equations for Bosons $\quad 326$

$27.2\left\langle n_{\epsilon}\right\rangle$ for Bosons $\quad 326$

27.3 The Ideal Bose Gas $\quad 327$

27.4 Low-Temperature Behavior of $\mu \quad 328$

27.5 Bose-Einstein Condensation $\quad 329$

27.6 Below the Einstein Temperature 330

27.7 Energy of an Ideal Gas of Bosons 331

27.8 What About the Second-Lowest Energy State? 332

27.9 The Pressure below $T<T_{E} \quad 333$

27.10 Transition Line in $P-V$ Plot $\quad 334$

27.11 Problems 334

28 Fermi-Dirac Statistics 336

28.1 Basic Equations for Fermions $\quad 336$

28.2 The Fermi Function and the Fermi Energy 337

28.3 A Useful Identity 338

28.4 Systems with a Discrete Energy Spectrum $\quad 339$

28.5 Systems with Continuous Energy Spectra $\quad 340$

28.6 Ideal Fermi Gas $\quad 340$

28.7 Fermi Energy $\quad 340$

28.8 Compressibility of Metals 341

28.9 Sommerfeld Expansion $\quad 342$

28.10 General Fermi Gas at Low Temperatures 345 
xiv Contents

28.11 Ideal Fermi Gas at Low Temperatures 346

28.12 Problems 348

29 Insulators and Semiconductors 351

29.1 Tight-Binding Approximation 351

29.2 Bloch's Theorem 353

29.3 Nearly-Free Electrons

29.4 Energy Bands and Energy Gaps $\quad 357$

29.5 Where is the Fermi Energy?

29.6 Fermi Energy in a Band (Metals) 358

29.7 Fermi Energy in a Gap 359

29.8 Intrinsic Semiconductors $\quad 362$

29.9 Extrinsic Semiconductors 362

29.10 Semiconductor Statistics 364

29.11 Semiconductor Physics $\quad 367$

30 Phase Transitions and the Ising Model 368

30.1 The Ising Chain 369

30.2 The Ising Chain in a Magnetic Field $(J=0) \quad 369$

30.3 The Ising Chain with $h=0$, but $J \neq 0$

30.4 The Ising Chain with both $J \neq 0$ and $h \neq 0 \quad 372$

30.5 Mean Field Approximation 376

30.6 Critical Exponents 380

30.7 Mean-Field Exponents 381

30.8 Analogy with the van der Waals Approximation $\quad 382$

30.9 Landau Theory $\quad 383$

30.10 Beyond Landau Theory 384

30.11 Problems $\quad 385$

Appendix: Computer Calculations and VPython 390

A.1 Histograms 390

A.2 The First VPython Program 391

A.3 VPython Functions $\quad 393$

A.4 Graphs 393

A.5 Reporting VPython Results 395

A.6 Timing Your Program $\quad 397$

A.7 Molecular Dynamics $\quad 397$

$\begin{array}{lll}\text { A.8 Courage } & 398\end{array}$

$\begin{array}{ll}\text { Index } & 399\end{array}$ 